\title{
Selective Excitation of High Order Modes in Few Mode Fibres Using Optical Microfibres
}

\author{
Bernard Oduro, Rand Ismaeel, Timothy Lee and Gilberto Brambilla \\ Optoelectronics Research Centre, University of Southampton, Southampton SO17 1BJ, United Kingdom \\ E-mail address: gilberto@soton.ac.uk
}

\begin{abstract}
Selective modal multiplexing and demultiplexing is demonstrated in few-mode fibers using weakly-fused microfiber couplers.
\end{abstract}

OCIS codes: (230.1150) All-optical devices; (060.1810) Buffers, couplers, routers, switches, and multiplexers.

\section{Introduction}

The continuously increasing demand in data transmission capacity has led to the investigation of alternative ways to augment the capacity of optical fiber communication systems, including multicore fibers, coherent communications, polarization and modal multiplexing [1]. In the mode division multiplexing (MDM), fibers, amplifiers and modal multiplexers have been promptly developed; various methods have been investigated to generate higher order modes and couple signals from single mode to selected high order mode, including the photonic lantern, which seems the most promising approach for simultaneous combination of numerous modes for modal division multiplexing [2].

A coupler made by weakly-fused optical fibers is proposed to solve the issue of adding/dropping single channels from MDM systems. Couplers have been manufactured from a single mode fiber (SMF) and a few-mode fiber (FMF) allowing for the efficient, virtually lossless, conversion of the fundamental mode at the SMF input to a selected high order mode at the FMF output.

\section{The weakly-fused optical fiber coupler}

Fig. 1(a) shows a weakly-fused intermodal coupler composed of a pre-tapered SMF (microfiber) thermally tacked to a FMF. Unlike conventional couplers, where the high processing temperature leads to the complete fusing between the fiberized coupler components and to an overall circular cross section, the low-temperature processing used in the weakly-fused coupler manufacture allows for the fusion of the two microfibers without any change at the cross section of the fiber components (fig. 1(b)).

(a)

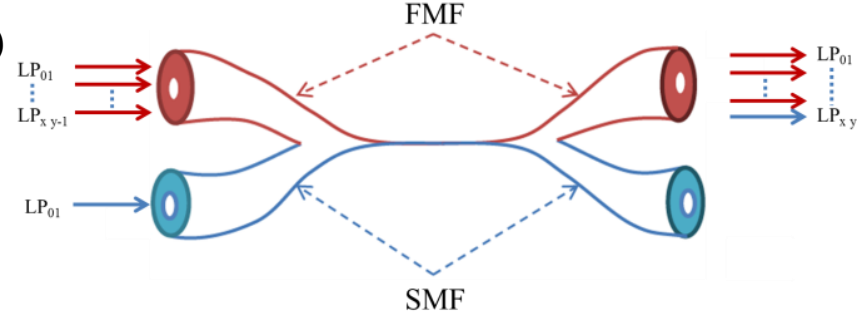

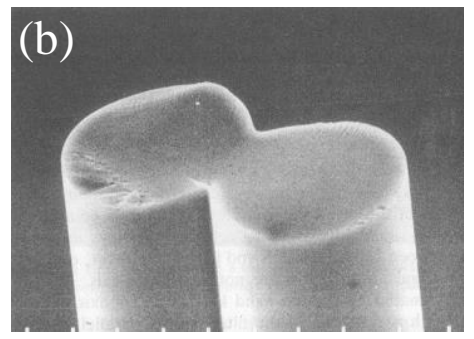

Fig. 1. (a) Schematic of a multimodal multiplexer using a few mode fiber (FMF) and a single mode fiber (SMF). The channel to be added is launched in the fundamental mode in the SMF and exits the device as a high order mode $\left(\mathrm{LP}_{\mathrm{xy}}\right)$ in the FMF. (b) SEM image of the coupler cross section showing weakly-fused optical microfibers.

Coupling (thus power exchange) between the SMF fundamental mode and the FMF high order mode is achieved by matching the propagation constants of the selected modes in the two fibers [3]. As the FMF has larger numerical aperture and core diameter than the SMF, all modes propagating in it have effective index higher than the modes propagating in the SMF. In silica optical microfibers with diameter smaller than $10 \mu \mathrm{m}$, the modal effective index is determined by the microfiber diameter, as modes are guided by the silica to air interface and are unaffected by the core size and its numerical aperture. Higher order modes are associated with lower effective indices (fig. 2) and matching of propagation constants of different modes can be achieved by using different microfiber diameters. In the coupler, intermodal phase matching is easily achieved with asymmetric cross sections. The coupling strength between different modes is inversely proportional to the mismatch between the mode propagation constants, meaning that coupling effectively takes place mostly between the mode of the microfiber manufactured from the SMF and the mode with closest propagation constant in the microfiber fabricated from the FMF. 


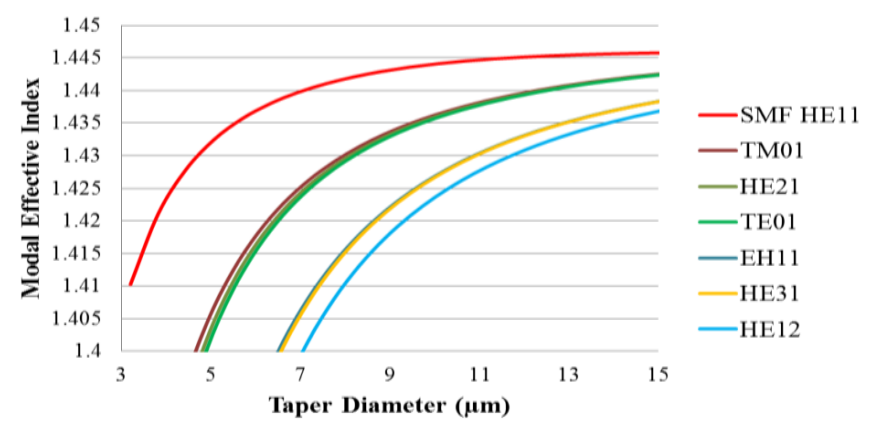

Fig. 2. Effective refractive index of modes supported by silica optical microfibers in air.

\section{Device fabrication and characterization}

Weakly-fused couplers have been manufactured using the modified flame brushing technique and a ceramic microheater (NTT-AT, Japan) [4]. While the SMF used in these experiments was a commercially available telecom fiber with cut-off at $\lambda=1.25 \mu \mathrm{m}$, different types of FMF have been used, including a two-mode and a four-mode fiber, all with outer diameter of $\mathrm{OD}=125 \mu \mathrm{m}$. The average insertion loss recorded in the couplers was of the order of $0.1 \mathrm{~dB}$. The SMF was pretapered to a set diameter determined by the desired final diameter ratio between the two different microfibers of the coupler. The ratio between the microfibers can be in first approximation obtained from fig. 2, which reports the eigenvalues of the propagation equation for a silica strand in air, assuming that the modes have the same modal effective index in the coupling region. The device length is optimized to have complete power transfer, from the SMF input to the FMF output in the add configuration and from the FMF input to the SMF output in the drop configuration. Examples of excitation of selected high order modes are presented in fig. 3 when no other mode is propagating in the FMF.
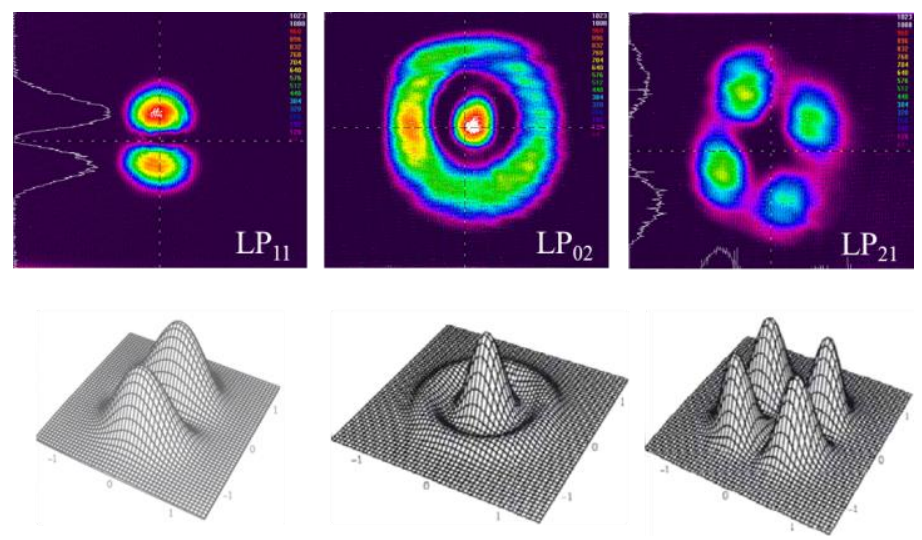

Fig. 3. Experimental (top) and simulated (bottom) intensity distribution for pure high order modes in a FMF. In the experimental results light was launched at $\lambda=1.55 \mu \mathrm{m}$ in the SMF input and collected from the FMF output.

The spectral purity has been evaluated using simple intensity level comparison between the maximum and the center of the mode on the CCD camera, as well as from the tight bend technique, confirming that for higher order modes it is instrumentation limited and well in excess of $90 \%$. As couplers are intrinsically asymmetric, long length couplers have allowed for the modal separation of different polarizations that are usually degenerate in a FMF.

\section{References}

[1] D. J. Richardson, J. M. Fini and L. E. Nelson, “Space-division multiplexing in optical fibres,” Nature Phot. 7, 354-362 (2013).

[2] S. Leon-Saval, N. Fontaine, J. Salazar-Gil, B. Ercan, R. Ryf, and J. Bland-Hawthorn, "Mode-selective photonic lanterns for space-division multiplexing," Opt. Express 22, 1036-1044 (2014).

[3] R. Ismaeel, T. Lee, B. Oduro, Y. Jung and G. Brambilla "All-fiber fused directional coupler for highly efficient spatial mode conversion," Opt. Express 22, 11610-11619 (2014).

[4] G. Brambilla, F. Koizumi, X. Feng and D.J. Richardson, “Compound-glass optical nanowires,” Electron. Lett. 41, 401-402 (2005). . 